Pesq. Vet. Bras. 30(8):618-622, agosto 2010

\title{
Correlação entre os métodos de concepção, ocorrência e formas de tratamento das onfalopatias em bovinos: estudo retrospectivo ${ }^{1}$
}

\author{
Celso A. Rodrigues ${ }^{2}$, Paulo Sergio P. dos Santos ${ }^{3}$, Sílvia Helena V. Perri ${ }^{4}$, \\ Piero Henrique M. Teodoro ${ }^{5}$, Carolina R. Anhesini ${ }^{5}$, Marcelo A. de Araújo ${ }^{6}$ \\ e Mário Nelson V. Filho ${ }^{7}$
}

\begin{abstract}
Rodrigues C.A., Santos P.S.P., Perri S.H.V., Teodoro P.H.M., Anhesini C.R., Araújo M.A.. \& Viana Filho M.N. 2010. [Correlation between conception methods, occurrence, and type o treatment of the umbilical diseases in cattle: A retrospective study.] Correlação entre os métodos de concepção, ocorrência e formas de tratamento das onfalopatias em bovinos: estudo retrospectivo. Pesquisa Veterinária Brasileira 30(8):618-622. Departamento de Cirurgia e Anestesiologia Veterinária, Faculdade de Medicina Veterinária e Zootecnia, Universidade Estadual Paulista "Júlio de Mesquita Filho" (Unesp), Campus de Botucatu, Distrito de Rubião Júnior s/n, Cx. Postal 560, Botucatu SP 18618-970, Brazil. E-mail: rodriguesca@ fmvz.unesp.br

Some problems have been observed in bovine products of the in vitro fertilization technical, among these, the high umbilical diseases casuistry. From this observation, the aim of this work was to accomplish a retrospective study of the correlation between the conception methods and the occurrence of umbilical diseases in bovines and describe the results obtained from the conservative and surgical treatments. For this 44 animals attended at Veterinary Teaching Hospital of Unesp Araçatuba, with age varying from one day to 12 months among the years of 2003 and 2007 were used, twenty seven were from in vitro fertilization (IVF), twelve were from artificial insemination (Al), two were from natural mounts and three were from embryo transfer. The clinical-surgical diagnosis of the animals revealed that all of them presented umbilical disorders, being 22 cases of urachus patent, eight omphalophlebitis, eight umbilical hernia, five omphalitis and one umbilical fibrosis. Before and at the postoperative period was administered in all animals once a day for ten days $3 \mathrm{mg} / \mathrm{kg}$ of ceftiofur sodium IV. In cases of serious infection or lack of response of the initial antimicrobial therapy a complementary treatment during seven days with $6.6 \mathrm{mg} / \mathrm{kg}$ of gentamicin sulfate IV was carried out. The antisepsis of the umbilicus with tincture of iodine to $2 \%$ was carried out twice daily in cases treated clinically, with out surgery, while the animals underwent surgery received $1.1 \mathrm{mg} / \mathrm{kg}$ of flunixin meglumine IV once daily for five consecutive days.Among the 22 animals diagnosed with urachus patent, 10 had urine drainage from the umbilicus and received $2 \mathrm{~mL}$ of $10 \%$ tincture of iodine in the urachus, and 15 was treated with surgical excision, due to formation of diverticulum from the blader to urachus. All animals that were affected with omphalophlebitis and umbilical hernia were submitted to surgery and already of the five cases of omphalitis, three were treated clinically. The analysis of different conception methods correlated to the umbilical diseases occurrence showed evidences that in this retrospective study, the animals from IVF have a higher frequency of the urachus patent
\end{abstract}

\footnotetext{
${ }^{1}$ Recebido em 13 de agosto de 2009.

Aceito para publicação em 8 de marco de 2010.

2 Departamento de Cirurgia e Anestesiologia Veterinária, Faculdade de Medicina Veterinária e Zootecnia (FMVZ), Universidade Estadual Paulista "Júlio de Mesquita Filho" (Unesp), Campus de Botucatu, Distrito de Rubião Júnior s/n, Caixa Postal 560, Botucatu SP 18618-970, Brazil. * Autor para correspondência: rodriguesca@fmvz.unesp.br

${ }^{3}$ Departamento de Clínica, Cirurgia e Reprodução Animal, Curso de
}

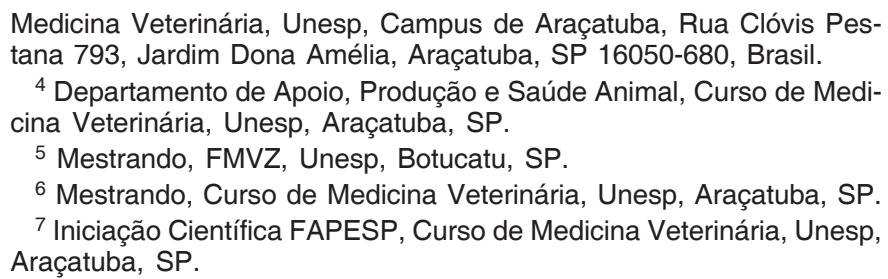

Medicina Veterinária, Unesp, Campus de Araçatuba, Rua Clóvis Pes. cina Veterinária, Unesp, Araçatuba, SP.

${ }^{6}$ Mestrando, Curso de Medicina Veterinária, Unesp, Araçatuba, SP. Araçatuba, SP. 
(66.7\%), and animals designed to Al observed higher frequency of umbilical hernia (58.4\%). There is high correlation between the IVF and the occurrence of umbilical diseases in calves and the conservative therapy was effective in less complicated cases and quickly identified, while the surgical treatment showed better results in the cases studied.

INDEX TERMS: Bovine, urachus patent, onfalitis, failure immunity transfer, in vitro fertilization.

RESUMO.- Alguns problemas têm sido observados nos bezerros produtos da técnica fertilização in vitro, dentre esses a elevada casuística de onfalopatias. A partir dessa observação, objetivou-se com este trabalho realizar um estudo retrospectivo da correlação entre os métodos de concepção e a ocorrência de onfalopatias em bovinos e descrever os resultados obtidos a partir dos tratamentos conservativo e cirúrgico. Foram utilizados 44 bovinos atendidos no Hospital Veterinário da Unesp, Campus de Araçatuba, com idade variando de um dia 12 meses entre os anos de 2003 e2007. Desses bovinos 27 eram provenientes de fertilização in vitro (FIV), 12 de inseminação artificial (IA), dois de monta natural (MN) e três de transferência de embriões (TE). O diagnóstico clínico-cirúrgico revelou que todos apresentavam afecções umbilicais, sendo 22 casos de persistência de úraco, oito de onfaloflebite, oito de hérnias umbilicais, cinco de onfalites e um de fibrose umbilical. Inicialmente e no pós-operatório administrou-se em todos os animais, uma vez ao dia, durante dez dias, 3mg/kg de ceftiofur sódico pela via intravenosa (IV). Nos casos de infecção grave ou irresponsiva a terapia antimicrobiana inicial, acrescentou-se $6,6 \mathrm{mg} / \mathrm{kg}$, durante sete dias de sulfato de gentamicina IV. A antissepsia do umbigo, com tintura de iodo a $2 \%$, foi instituída duas vezes ao dia, nos casos tratados conservativamente, enquanto que os bovinos submetidos à cirurgia receberam $1,1 \mathrm{mg} / \mathrm{kg}$ de flunixin meglumine IV, uma vez ao dia, por cinco dias consecutivos. Dos 22 animais diagnosticados com persistência de úraco, 10 apresentavam drenagem de urina pelo umbigo e receberam $2 \mathrm{~mL}$ de tintura de iodo $10 \%$ no interior do úraco, sendo 15 tratados com a excisão cirúrgica, especialmente, devido à formação de divertículo vésico-uracal. Todos os animais que apresentavam onfaloflebite e hérnia umbilical foram submetidos à cirurgia. Já dos cinco casos de onfalite, três foram tratados conservativamente. A análise dos diferentes métodos de concepção, correlacionados à ocorrência de onfalopatias, sugere que os animais provenientes de FIV, apresentam maior frequência de persistência de úraco $(66,7 \%)$, e aqueles concebidos por IA, maior frequência de hérnia umbilical $(58,4 \%)$, O tratamento cirúrgico foi mais eficiente que a terapia conservativa. Essa última apresentou melhores resultados nos casos descomplicados e precocemente diagnosticados.

TERMOS DE INDEXAÇÃO: Bovino, úraco patente, onfalites, falha na transferência da imunidade, fertilização in vitro.

\section{INTRODUÇÃO}

A elevada taxa de mortalidade neonatal em animais pecuários se deve as dificuldades frequentemente enfrentadas pelos recém-nascidos durante a fase de adaptação à vida pós-natal (Benesi 1993, Donovan et al. 1998, Feitosa et al. 2001, Smith 2006). Dentre as adversidades apontadas, a inadequada trans- ferência de imunidade passiva torna o neonato um alvo fácil para as infecções bacterianas e virais, tornando a ocorrência dessas, dependentes do balanço entre a imunidade passiva adquirida e o desafio provocado pelo meio ambiente (White 1993).

A falha de transferência passiva das imunoglobulinas do colostro e as práticas de manejo com higiene deficiente são os maiores determinantes de mortalidade dos bezerros (Walther et al. 1986, Donovan et al. 1998). Desta forma, minimizar a exposição aos patógenos é certamente um dos métodos mais fáceis e de melhor custo benefício para aumentar o índice de sobrevivência desses animais (Waltner et al. 1986). A deficiente transferência passiva de imunoglobulinas seja por abandono materno ou incapacidade de mamar leva à inanição e aumento da suscetibilidade a infecções, com efeitos sobre a taxa de mortalidade e incidência de enfermidades como colibacilose, poliartrites, septicemia e a maioria das infecções entéricas virais no período compreendido entre dois e sete dias de idade (Feitosa et al. 2001). O animal neonato frequentemente apresenta desafios diagnósticos e terapêuticos e, por esse motivo, o diagnóstico e o tratamento devem ser consideravelmente acurados e rápidos (Radostits et al. 2002, Rengifo et al. 2006).

A infecção das estruturas umbilicais é comum em animais com falha na imunidade passiva, e pode resultar em bacteremia, septicemia e morte em neonatos. Microrganismos usuais de onfalite são frequentemente isolados em animais com bacteremia e septicemia, comprovando que as estruturas umbilicais são importantes portas de entrada para agentes causadores destas enfermidades (Hathaway et al. 1993, Rebhum 1995, Ogilvie 2000, Radostits et al. 2002, Rengifo et al. 2006). A falta de higiene, negligência nos primeiros cuidados, soluções antissépticas contaminadas, produtos inadequados e ou mal aplicados após o nascimento, também são fatores predisponentes às afecções umbilicais (Radostits et al. 2002). O tratamento das afecções umbilicais pode ser conduzido de modo conservativo, com a utilização de antissépticos e antimicrobianos, porém, dificilmente ocorre a cura, enquanto que a cirurgia, objetivando a extirpação completa das estruturas acometidas é o método de eleição para o tratamento dessas enfermidades (Figueirêdo 1999).

A aspiração folicular guiada por ultrassonografia e posterior fertilização in vitro dos oócitos, obtidos de doadoras vivas e de alto valor genético é uma técnica amplamente utilizada no manejo reprodutivo de bovinos, entretanto, alguns problemas têm sido observados nos animais produtos desta técnica, incluindo aumento do período de gestação, elevação da mortalidade perinatal, maior proporção de machos, edema fetal, alterações no desenvolvimento de órgãos como coração, cérebro, coluna espinhal e músculos esqueléticos, alterações de parâmetros bioquímicos, falhas no desenvolvimento das membranas córion alantóide e vasos sanguíneos, 
alterações na morfologia dos placentomas e contato materno fetal e aumento da ocorrência de hidroalantóide e hidroâmnio. Esses problemas, possivelmente, seriam causados por uma falha no padrão de impressão e expressão de genes que regulam tais mecanismos (Farin et al. 2006).

O objetivo deste estudo foi realizar um estudo retrospectivo, correlacionando os métodos de concepção e a ocorrência de onfalopatias em bezerros, bem como descrever os resultados obtidos a partir dos tratamentos conservativo e cirúrgico.

\section{MATERIAL E MÉTODOS}

Foram utilizados neste estudo 44 animais, machos e fêmeas, com idade variando de um dia a 12 meses, sendo 33 da raça Nelore, seis Holandês, dois Brahman, dois mestiços e um Brangus, com historio de onfalopatias e encaminhados ao Hospital Veterinário da UNESP - Campus de Araçatuba - SP, entre os anos de 2003 a 2007. Desses bovinos, 27 eram provenientes de fertilização in vitro (FIV), 12 de inseminação artificial (IA), dois de monta natural (MN) e três por transferência de embriões (TE).

Preliminarmente foi realizado em todos os animais exame clínico geral, enfatizando os parâmetros de frequências cardíaca e respiratória, temperatura corpórea, coloração das membranas mucosas e turgor da pele. Nesse momento foram colhidas amostras de sangue dos bovinos para realização de hemograma e após o exame clínico geral procedeu-se o exame específico da região umbilical de todos os animais. Durante a inspeção verificou-se a presença ou ausência de aumento de volume na região, presença ou ausência de sensibilidade local, consistência, redutibilidade, bem como se esse aumento estava associado ou não a drenagem de pus e ou urina.

Os bezerros receberam tratamento de suporte no período pré, trans e pós-operatório baseado no exame clínico geral e hemograma, objetivando corrigir a desidratação e desequilíbrio ácido-básico. Previamente e no período pós-operatório administrou-se em todos os animais uma vez ao dia, $3 \mathrm{mg} / \mathrm{kg}$ de ceftiofur sódico IV, totalizando 10 aplicações, sendo que nos oito animais acometidos de onfaloflebite essa terapia antimicrobiana foi acrescida de $6,6 \mathrm{mg} / \mathrm{kg}$ de sulfato de gentamicina, durante sete dias. Adicionalmente também se procedeu a antissepsia do umbigo, com tintura de iodo a $2 \%$, duas vezes ao dia nos bovinos tratados conservativamente e com polivinil pirrolidona tópica $10 \%$, nos animais submetidos à cirurgia.

O teste de qui-quadrado com correção de continuidade de Yates, foi empregado para verificar a associação entre persistência do úraco e FIV. Posteriormente, foi utilizado o teste exato de Fisher para verificar a associação entre hérnia umbilical e IA e entre as demais variáveis do diagnóstico e a forma de concepção, bem como entre as variáveis: complicações e evolução e o tratamento. As estatísticas foram consideradas significativas quando $p<0,05$. As análises estatísticas foram efetuadas empregando-se o programa computacional $\mathrm{SAS}^{7}$.

\section{RESULTADOS E DISCUSSÃO}

O exame clínico específico dos bovinos revelou 22 casos de persistência de úraco, oito de onfaloflebite, oito de hérnias umbilicais, cinco de onfalites e um de fibrose umbili-

\footnotetext{
7 SAS Institute Inc., Version 8, SAS Institute Inc., Cary, 1999.
}

Quadro 1. Número (N) e porcentagem (\%) de bovinos $(n=44)$ acometidos por onfalopatias e distribuídos de acordo com as variáveis (raça, forma de concepção, diagnóstico, complicações, tratamento e evolução)

\begin{tabular}{lccc}
\hline \multirow{2}{*}{ Variável } & Categoria & $\mathrm{N}$ & $\%$ \\
\hline \multirow{4}{*}{ Foça } & Brahmam & 2 & 4,5 \\
& Brangus & 1 & 2,3 \\
& Holandês & 6 & 13,6 \\
& Nelore & 33 & 75,1 \\
& Mestiço & 2 & 4,5 \\
Diagnóstico de concepção & Fertilização in vitro & 27 & 61,4 \\
& Inseminação artificial & 12 & 27,3 \\
& Monta natural & 2 & 4,5 \\
& Transferência de embrião & 3 & 6,8 \\
Complicação & Fibrose umbilical & 1 & 2,3 \\
& Hérnia umbilical & 8 & 18,2 \\
& Onfalite & 5 & 11,4 \\
& Onfaloflebite & 8 & 18,2 \\
& Persistência de úraco & 22 & 49,9 \\
& Artrite & 1 & 2,3 \\
Tratamento & Eventração & 1 & 2,3 \\
Evolução & Hérnia umbilical & 4 & 9,1 \\
& Ruptura do úraco & 1 & 2,3 \\
& Septicemia & 2 & 4,5 \\
& Úlcera de abomaso & 2 & 4,5 \\
& Nenhuma & 33 & 75,0 \\
& Cirúrgico & 34 & 77,3 \\
& Conservativo & 10 & 22,7 \\
& Alta & 37 & 84,1 \\
& Óbito & 7 & 15,9
\end{tabular}

cal (Quadro 1). Dos 22 animais diagnosticados com persistência de úraco, 10 apresentavam drenagem de urina pelo umbigo e receberam $2 \mathrm{~mL}$ de tintura de iodo $10 \%$ no interior do úraco, sendo 13 tratados com a excisão cirúrgica, devido à formação de divertículo vésico-uracal. Posteriormente outros dois bezerros, tratados preliminarmente com tintura de iodo, foram encaminhados à cirurgia. Todos os animais que apresentavam onfaloflebite e hérnia umbilical foram submetidos à cirurgia, incluindo o caso de fibrose umbilical (17 animais). Já dos cinco casos de onfalite, três foram tratados conservativamente.

Dentre as onfalopatias apresentadas, a persistência de úraco associada à formação de divertículo vésico-uracal, apresentou maior incidência nos animais estudados. Embora relatado que a cura dessas enfermidades com o tratamento conservativo seja rara (Figueirêdo 1999), optouse por este tratamento em 10 animais, obtendo-se $50 \%$ de êxito, pois, cinco bezerros se recuperaram sem a necessidade de cirurgia e outros cinco apresentaram algum tipo de complicação, sendo que dois foram tratados cirurgicamente e três morreram (Quadros 3-4).

$\mathrm{O}$ tratamento conservativo se mostrou ineficiente em cinco animais, aonde três vieram a óbito, devido provavelmente ao grave quadro séptico e às complicações previamente instaladas e outros dois foram encaminhados à cirurgia. A mesma ineficiência do tratamento conservativo, devido às complicações anteriormente descritas, foi observada em três bezerros que apresentavam onfalite.

O tratamento cirúrgico foi realizado em 34 animais, sendo 16 decorrentes de persistência de úraco ou divertículo 
Quadro 2. Número $(\mathrm{N})$ e porcentagem (\%) de bovinos ( $n=44)$ acometidos por onfalopatias e distribuídos de acordo com o diagnóstico e a forma de concepção

\begin{tabular}{|c|c|c|c|c|c|c|c|c|c|c|}
\hline \multirow{3}{*}{ Diagnóstico } & \multicolumn{8}{|c|}{ Forma de concepção } & \multicolumn{2}{|c|}{ Total } \\
\hline & \multicolumn{2}{|c|}{ FIVa } & \multicolumn{2}{|c|}{$\mathrm{IA}^{\mathrm{b}}$} & \multicolumn{2}{|c|}{$\mathrm{MNC}^{\mathrm{C}}$} & \multicolumn{2}{|c|}{ TE $^{d}$} & \multirow[t]{2}{*}{$\mathrm{N}$} & \multirow[t]{2}{*}{$\%$} \\
\hline & $\mathrm{N}$ & $\%$ & $\mathrm{~N}$ & $\%$ & $\mathrm{~N}$ & $\%$ & $\mathrm{~N}$ & $\%$ & & \\
\hline Fibrose umbilical & 1 & 3,7 & - & - & - & - & - & - & 1 & 2,3 \\
\hline Hérnia umbilicale & 1 & 3,7 & 7 & 58,4 & - & - & - & - & 8 & 18,2 \\
\hline Onfalite & 2 & 7,4 & 1 & 8,3 & - & - & 2 & 66,7 & 5 & 114 \\
\hline Onfaloflebite & 5 & 18,5 & 3 & 25,0 & - & - & - & - & 8 & 18,2 \\
\hline Persistência de úraco ${ }^{f}$ & 18 & 66,7 & 1 & 8,3 & 2 & 100,0 & 1 & 33,3 & 22 & 49,9 \\
\hline Total & 27 & 100,0 & 12 & 100,0 & 2 & 100,0 & 3 & 100,0 & 44 & 100,0 \\
\hline
\end{tabular}

Quadro 3. Número (N) e porcentagem (\%) de bovinos $(n=44)$ acometidos por onfalopatias e distribuídos de acordo com as complicações observadas e o tratamento

\begin{tabular}{|c|c|c|c|c|c|c|}
\hline \multirow[t]{3}{*}{ Complicações } & \multicolumn{4}{|c|}{ Tratamento } & \multicolumn{2}{|c|}{ Total } \\
\hline & \multicolumn{2}{|c|}{ Cirúrgico } & \multicolumn{2}{|c|}{ Conservativo } & \multirow[t]{2}{*}{$\mathrm{N}$} & \multirow[t]{2}{*}{$\%$} \\
\hline & $\mathrm{N}$ & $\%$ & $\mathrm{~N}$ & $\%$ & & \\
\hline Artrite & 1 & 2,9 & - & - & 1 & 2,3 \\
\hline Eventração & 1 & 2,9 & - & - & 1 & 2,3 \\
\hline Hérnia umbilical & 4 & 11,8 & - & - & 4 & 9,1 \\
\hline Ruptura do úraco & 1 & 2,9 & - & - & 1 & 2,3 \\
\hline Septicemia & 1 & 2,9 & 1 & 10,0 & 2 & 4,5 \\
\hline Úlcera de abomaso & - & - & 2 & 20,0 & 2 & 4,5 \\
\hline Nenhuma & 26 & 76,6 & 7 & 70,0 & 33 & 75,0 \\
\hline Total & 34 & 100,0 & 10 & 100,0 & 44 & 100,0 \\
\hline
\end{tabular}

Teste exato de Fisher: $p=0,1395$.

vésico-uracal, oito hérnias umbilicais, oito onfaloflebites e duas onfalites, sendo que somente dois morreram (Quadro 4) devido à peritonite e úlcera de abomaso, sendo esta última verificada em quatro animais tratados.

A análise dos diferentes métodos de concepção, correlacionados à ocorrência de enfermidades umbilicais, demonstrou associação significativa entre a persistência de úraco $(66,7 \%)$ e a FIV $(p=0,0133)$, bem como entre a hérnia umbilical $(58,4$ $\%)$ e a IA ( $p=0,0001)$. As demais variáveis testadas não apresentaram associação estatisticamente significativa (Quadro 2). Esta correlação pode ser atribuída às falhas na transferência de imunidade passiva colostral ou de alterações proporcionadas pela técnica de concepção. Estas falhas podem ocorrer mesmo em criações em que as boas práticas de manejo são adotadas, pois, em algumas situações o parto dos animais ocorre durante o período noturno, não recebendo o neonato um acompanhamento satisfatório. Desta maneira, o bezerro não mama o volume de colostro adequado, ou o faz fora do período ideal de absorção das imunoglobulinas. A incapacidade do neonato em mamar o colostro, o momento em que é ingerido e a quantidade e/ou qualidade inadequadas, pode causar falha na transferência de imunidade passiva (McGuire \& Adams 1982, White 1993). A síndrome asfixia neonatal, que, acarreta uma menor vitalidade do recém-nascido, pode também retardar e diminuir a ingestão colostral tornando o animal susceptível a infecções oportunistas (Benesi 1993).

É importante ressaltar que as concentrações séricas de
Quadro 4. Número (N) e porcentagem (\%) de bovinos $(n=44)$ acometidos por onfalopatias e distribuídos de acordo com a evolução e o tratamento

\begin{tabular}{|c|c|c|c|c|c|c|}
\hline \multirow[t]{3}{*}{ Evolução } & \multicolumn{4}{|c|}{ Tratamento } & \multicolumn{2}{|c|}{ Total } \\
\hline & \multicolumn{2}{|c|}{ Cirúrgico } & \multicolumn{2}{|c|}{ Conservativo } & \multirow[t]{2}{*}{$\mathrm{N}$} & \multirow[t]{2}{*}{$\%$} \\
\hline & $\mathrm{N}$ & $\%$ & $\mathrm{~N}$ & $\%$ & & \\
\hline Alta & 32 & 94,1 & 5 & 50,0 & 37 & 84,1 \\
\hline Morte & 2 & 5,9 & 5 & 50,0 & 7 & 15,9 \\
\hline Total & 34 & 100,0 & 10 & 100,0 & 44 & 100,0 \\
\hline
\end{tabular}

Teste exato de Fisher: $p=0,0039$.

imunoglobulinas estão altamente correlacionadas com as quantidades ingeridas na primeira alimentação, de tal forma que, um colostro com baixa quantidade de imunoglobulinas, irá acarretar uma inadequada transferência de imunidade passiva (Aldridge et al. 1992). Entretanto, não foram realizadas mensurações das concentrações séricas de imunoglobulinas no momento do exame clínico e especialmente ao nascimento, que reportem a qualidade da transferência de imunidade nos animais estudados. Além disso, para estabelecer correlação entre as onfalopatias, falha de transferência de imunidade passiva e FIV, deveriam ser avaliadas diversas populações a partir do nascimento.

Os animais provenientes de FIV estudados possuíam elevado valor comercial e foram encaminhados para tratamento no Hospital Veterinário, quando da verificação de alterações umbilicais, sendo esse encaminhamento realizado até 12 meses após o nascimento. Dessa forma, mesmo que análise das imunoglobulinas tivesse sido realizada, somente demonstraria o status imunológico momentâneo e não durante a fase neonatal, referenciada como das mais importantes (Benesi 1993, Feitosa et al. 2001).

A hipoimunoglobulinemia pode não ser a única explicação para a elevada incidência de onfalopatias em animais concebidos por FIV. Uma vez que certas afecções têm sido frequentemente observadas nos animais produtos desta técnica, incluindo alterações de parâmetros bioquímicos, falhas no desenvolvimento das membranas córion alantóide e vasos sanguíneos, alterações na morfologia dos placentomas e contato materno fetal. Sendo essas alterações possivelmente causadas por uma falha no padrão de impressão e expressão de genes que regulam tais mecanismos (Farin et al. 2006). 
Mostra-se a maior frequência de persitência de úraco (Quadro 2) em animais provenientes de FIV, correlacionando a prevalência dessas com o método de concepção, como relatado por Farin et al. (2006), que hipotetizaram falhas nos mecanismos fisiológicos genéticos essenciais para o desenvolvimento fetal e pós-natal à técnica de FIV. A suposição de falhas genéticas, durante o processo de FIV, tem motivado estudos para identificação precisa dessa alteração indesejável que após avaliação mais detalhada possibilitarão observar se tal falha de transferência ocorre, ou se a biotécnica de FIV induz a alguma deficiência no sistema imune do recém-nascido. Assim, a ocorrência de onfalopatias, pode apresentar origem multifatorial em animais concebidos por FIV devido a defeitos congênitos, retração do cordão umbilical, impressão e expressão gênica, devendo as falhas na transferência de imunidade passiva, investigada a partir do nascimento e comparada entre as populações sadias e acometidas. Porém, essa hipótese necessita de comprovação científica.

\section{CONCLUSÕES}

A análise dos diferentes métodos de concepção, correlacionados à ocorrência de onfalopatias, sugere que os animais provenientes de FIV, apresentam maior frequência de persistência de úraco $(66,7 \%)$, e aqueles concebidos por IA, apresentam maior frequência de hérnia umbilical $(58,4 \%)$,

O tratamento cirúrgico foi mais eficiente que a terapia conservativa, sendo que essa última apresentou melhores resultados nos casos descomplicados e precocemente diagnosticados.

Agradecimentos. - À Fundação de Amparo à Pesquisa do Estado de São Paulo (FAPESP) proc.2008/00702-7, pela concessão de bolsa de iniciação científica e à Fundação Para o Desenvolvimento da UNESP (FUNDUNESP) pelos recursos para publicação.

\section{REFERÊNCIAS}

Aldridge B., Garry F. \& Adams R. 1992. Role of colostral transfer in neonatal calf management: Failure of aquisition of passive immunity. Compend. Cont. Educ. Vet. Pract. 14(2):265-270.
Benesi F.J. 1993. Síndrome asfixia neonatal nos bezerros: importância e avaliação crítica. Arqs Esc. Med. Vet. Univ. Fed. Bahia 16:3848.

Donovan G.A., Dohoo R.I., Montgomery D.M. \& Bennett F.L. 1998. Cattle morbidity and mortality: Passive immunity. Prev. Vet. Med. 34(1):31-46.

Farin P.W., Piedrahita J.A. \& Farin C.E. 2006. Errors in development of fetuses and placentas from in vitro-produced bovine embryos. Theriogenology 65:178-191.

Figueirêdo L.J.C. 1999. Onfalopatias de bezerros. Editora da Universidade Federal da Bahia, Salvador. 94p.

Feitosa F.L.F., Birgel E.H., Mirandola R.M.S. \& Perri S.H.V. 2001. Diagnóstico de falha de transferência de imunidade passiva em bezerros através da determinação de proteína total e de suas frações eletroforéticas, imunoglobulinas $\mathrm{g}$ e $\mathrm{m}$ e da atividade da gama glutamil transferase no soro sanguíneo. Ciência Rural 31(2):251255.

Hathaway S.C., Bullians J.A., Johnstone A.C., Biss M.E. \& Thompson A. 1993. A pathological and microbiological omphalophlebitis in very young calvies slaughtered in New-Zealand. N. Z. Vet. J. 41:166170.

McGuire T.C. \& Adams D.S. 1982. Failure of colostral immunoglobulin transfer to calves: Prevalence and diagnosis. Cont. Educ. Article 4(1):35-40.

Ogilvie T.H. 2000. Medicina Interna de Grandes Animais. Artes Médicas Sul, Porto Alegre, p.468-470.

Radostits O.M., Gay C.C., Blood D.C. \& Hinchcliff K.W. 2002. Clinica veterinária: um tratado de doenças dos bovinos, ovinos, suínos, caprinos e eqüinos. 9 ${ }^{a}$ ed. Guanabara Koogan, Rio de Janeiro, p.102-136.

Rebhum W.C. 1995. Urinary tract diseases, p.365-366. In: Ibid. (Ed.), Diseases of Dairy Cattle. Williams and Wilkins, Baltimore.

Rengifo S.A., Silva R.A., Pereira I.A., Zegarra J.Q., Souza M.M. \& Botteon R.C.C.M. 2006. Isolamento de agentes microbianos a partir de amostras de sangue e umbigo de bezerros mestiços neonatos. Braz. J. Vet. Res. Anim. Sci. 43(4):442-447.

Smith B.P. 2006. Medicina Interna de Grandes Animais. $3^{\text {a }}$ ed. Manole, Barueri, p.255-386.

Waltner T.D., Martin S.W. \& Meek A.H. 1986. An epidemiological study of selected calf pathogens on holstein dairy farms in Southwestern Ontario. Can. J. Vet. Res. 50:307-313.

White D.G. 1993. Colostral suplementation in ruminants. Compend. Cont. Educ. Vet. Pract. 15:335-342. 